\title{
REDUNDANCY MODULATION FOR COHERENT IMAGING SYSTEMS $\dagger$
}

\author{
R.W. LEWIS \\ Radar and Optics Laboratory, The University of Michigan, Ann Arbor, Michigan, USA
}

Received 19 October 1972

\begin{abstract}
In order to image continuous-tone transparencies with coherent light, special types of diffuse speckle-free illumination are required to achieve high image quality. Several types of speckle-free modulation have been investigated which appear to provide high redundancy for such systems.
\end{abstract}

When continuous-tone transparencies are coherently imaged, optical noise such as dust diffraction patterns can degrade the image quality. Diffusers or gratings have been used to increase the spatial bandwidth of the signal in order to introduce redundancy into coherent imaging systems and to reduce noise effects $[1,2]$. It is desirable that redundancy modulation have a uniform power spectrum in order to evenly disperse noise diffraction patterns at the image plane. Several classes of periodic, speckle-free, band-limited modulation with good redundancy characteristics have been analyzed and are described here.

First to be considered is a type of phase modulation which under certain conditions has a rather uniform, band-limited power spectrum. Let $q(x) s(x)$ be the complex input signal amplitude for a band-limited coherent imaging system, where $s(x)$ is the amplitude transmittance of a continuous-tone transparency and $q(x)$ represents complex modulation used to increase the spatial bandwidth of the transparency. A logical function $q(x)$ to consider is a train of quadratic phase signals,

$q_{\mathrm{A}}(x)=\left[\exp \left( \pm \pi \mathrm{i} x^{2} / \lambda X F\right) \operatorname{rect}(x / X)\right] * \sum_{n=-\infty}^{\infty} \delta(x-n X)$,

where

$$
\begin{aligned}
\operatorname{rect}(x) & \equiv 1 \text { for }|x| \leqslant \frac{1}{2}, \\
& \equiv 0 \text { otherwise. }
\end{aligned}
$$

$\dagger$ This work was supported by NSF Grant GK-31474. $\delta$ denotes the Dirac delta function. It is well known that such a signal has a band-limited uniform spectral density if the space handwidth parameter $Q=X / \lambda F$ $\gg 10[3,4]$. If the maximum phase variation $\frac{1}{4} \pi Q$ is not too large, the modulation $q_{\mathrm{A}}(x)$ can be realized using a fly's eye lens array consisting of a train of positive or negative lenslets. The discontinuity where the lenslets join can be removed if a new function

$$
\begin{aligned}
& q_{\mathrm{B}}(x)=\left\{\exp \left(\frac{1}{4} \pi \mathrm{i} Q\right) \exp \left(-\pi \mathrm{i} x^{2} / \lambda X F\right) \operatorname{rect}(x / X)\right. \\
& \left.\quad+\exp \left(-\frac{1}{4} \pi \mathrm{i} Q\right) \exp \left[\pi \mathrm{i}(x-X)^{2} / \lambda X F\right] \operatorname{rect}[(x-X) / X]\right\} \\
& \quad * \sum_{n=-\infty}^{\infty} \delta(x-2 n X)
\end{aligned}
$$

is considered with alternating positive and negative lenslets. If $\hat{q}_{\mathrm{A}}(n / X)$ and $\hat{q}_{\mathrm{B}}(n / 2 X)$ represent the $n$th complex Fourier series coefficient of $q_{\mathrm{A}}(x)$ and $q_{\mathrm{B}}(x)$ respectively, it can easily be shown using superposition that

$$
\begin{aligned}
\hat{q}_{\mathrm{B}}(n / 2 X) & =2 \operatorname{Re}\left\{\exp \left(\frac{1}{4} \pi \mathrm{i} Q\right) \hat{q}_{\mathrm{A}}(n / 2 X)\right\}, n \text { even, } \\
& =2 \operatorname{Im}\left\{\exp \left(\frac{1}{4} \pi \mathrm{i} Q\right) \hat{q}_{\mathrm{A}}(n / 2 X)\right\}, n \text { odd }
\end{aligned}
$$

Experiments were performed showing the noise reduction characteristics of $q_{\mathrm{B}}(x)$ in a coherent imaging system. Fig. 1 shows a coherent imaging system where the function $q_{\mathrm{B}}\left(y_{1}\right)$ was used to introduce re- 


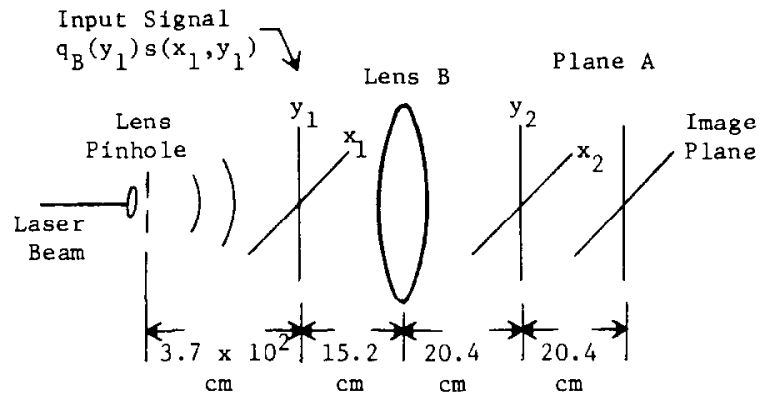

Fig. 1. Coherent imaging system where lens $B$ has a focal length of $100 \mathrm{~mm}$ and an F-number of 2.3 . Two wires each $0.23 \mathrm{~mm}$ in diameter were introduced at plane $\mathrm{A}$.

dundancy along the $y_{1}$ coordinate; $X, Q$, and $\lambda$ for $q_{\mathrm{B}}\left(y_{1}\right)$ have values $0.5 \mathrm{~mm}, 142$ and $0.6328 \mu \mathrm{m}$ respectively. Since there exists no redundancy along the $x_{1}$-signal coordinate two wires placed in plane A parallel to the $y_{2}$ axis introduce dark diffraction patterns at the image plane as shown in fig. 2a. The maximum number of cycles per mm of the signal bar pattern is 7. However, when the two wires are placed parallel to the $x_{2}$ axis, the wire diffraction patterns are highly attenuated as shown in fig. 2 b. Fig. 3 shows the irradiance at the back focal plane of lens B. $q_{\mathrm{B}}\left(y_{1}\right)$ had a spatial bandwid th of 284 cycles per $\mathrm{mm}$.

For certain coherent imaging constraints, modulation such as $q_{\mathrm{A}}(x)$ or $q_{\mathrm{B}}(x)$ cannot provide adequate redundancy. For example, when redundancy modulation $q(x)$ with period $X$ is used to introduce redundancy into a high-density holographic storage system, constraints on the system bandwidth $W$ and the period $X$

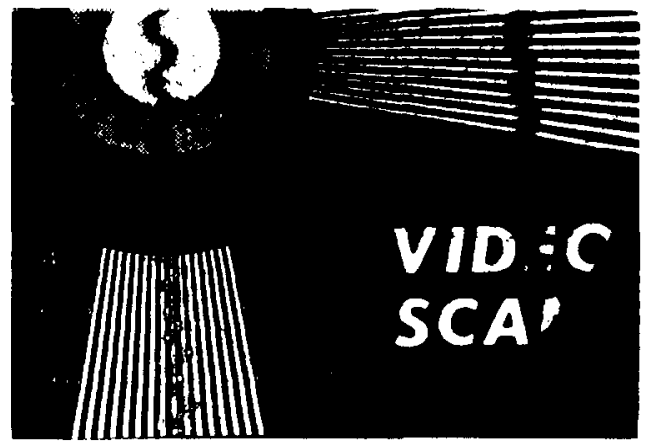

(a)

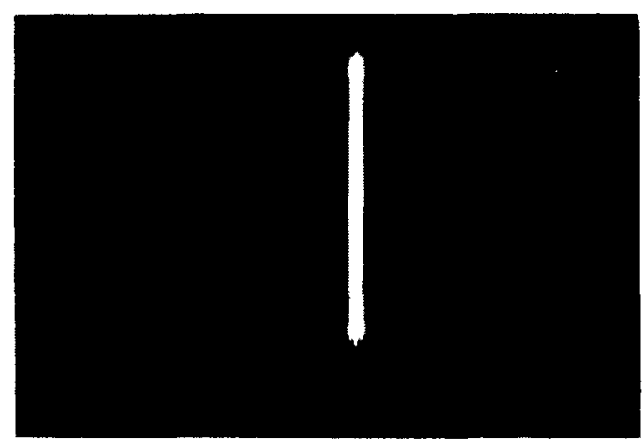

Fig. 3. Irradiance near the back focal plane of lens B.

place an upper limit on the space bandwidth product $X W$ and thus on the parameter $Q$. If $Q$ is small, the spectral density of a lens array is no longer uniform.

The type of speckle free periodic modulation proposed by Gabor [5] can be used to achieve high redundancy when the space bandwidth product $X \mathrm{~W}$ must be low. Gabor investigated the class of bandlimited functions:

$$
q(x)=\sum_{n=-N}^{N} a_{n} \exp (2 \pi \mathrm{i} n x / X) .
$$

Since

$$
|q(x)|^{2}=I_{0}+2 \sum_{n=1}^{2 N} I_{n} \cos \left(2 \pi n x / X+\phi_{n}\right),
$$

where

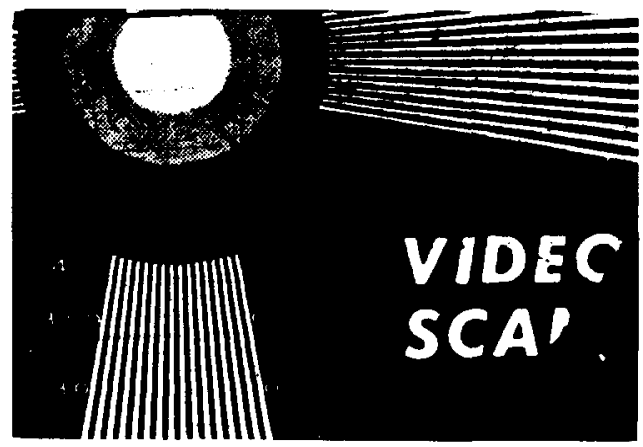

(b)

Fig. 2, a) Coherent image with two vertical wires placed in plane A of fig. 1. b) Coherent image with two horizontal wires placed in plane $A$ of fig. 1 , whose vertical positions are indicated with arrows. 
$I_{k}=\left|\sum_{n=-N}^{N-k} a_{n} a_{n+k}^{*}\right|$

low frequency speckle modulation is eliminated if the system of nonlinear equations given below is satisfied.

$\sum_{n=-N}^{N-k} a_{n} a_{n+k}^{*}=0$ for $k=1,2, \ldots, 2 N-2,2 N-1$.

Since only the relative values of the coefficients $\left\{a_{n}\right\}_{n=-N}^{N}$ are of interest, let $a_{N}=i$. Assuming that only even functions are of interest, $a_{-n}=a_{n}$, and the system of equations ( 1 ) above reduces to $2 N-1$ equations and $2 N$ unknowns. This leaves one free parameter which can be varied in order to maximize the redundancy parameter $R_{\mathrm{M}}$ defined as

$R_{\mathrm{M}} \equiv \sum_{n=-N}^{N}\left|a_{n}\right|^{2} / \max _{n}\left(\sum_{j=1}^{M}\left|a_{n+j}\right|^{2}\right)$,

in order to obtain a grating with the best power spectrum uniformity. The class of solutions obtained by Gabor possessed the $90^{\circ}$ phase property, arg $\left\{a_{n} a_{n+1}^{*}\right\}$ $= \pm \frac{1}{2} \pi$, where $\arg \{\because\}$ denotes the phase of a complex number. The phase of a solution $q(x)=m(x) \exp [\mathrm{i} P(x)]$ with this property satisfies the equation $P\left(x+\frac{1}{2} X\right)+C$ $=-[P(x)+C]$ (modulo $2 \pi$ ), where $C$ is a real constant. It was found that the recurrence relationship given by Gabor to generate solutions to eqs. (1) is not valid for $N>3$. Solutions for $N>3$ do exist, however, and have been calculated for $N=4,5,6$. The solutions obtained for $N=5$ and 6 assumed $\arg \left\{a_{n} a_{n+1}^{*}\right\}= \pm \frac{1}{2} \pi$ since this property simplifies eqs. (1) and makes each equation of (1) represent the addition of collinear vectors. If the $90^{\circ}$ phase condition is not imposed, solutions to eqs. (1) are more difficult to obtain, but solutions with higher redundancy can be found.

Table 1 lists solutions $\left\{a_{n}\right\}_{n=-N}^{N}$ to eqs. (1) which maximize the redundancy parameter $R_{1}$. Let FT $\{\cdot\}$ denote the Fourier transform operation. Figs. 4 and 5 show the coefficients $|\hat{q}(n / X)|^{2}=\left|a_{n}\right|^{2}$ and the envelope $|\mathrm{FT}\{\operatorname{rect}(x / X) q(x)\}|^{2}$ for entries $\mathrm{E}$ and $\mathrm{F}$ in table 1. Fig. 6 shows the phase $P(x)=\arg \{q(x)\}$ for entry $F$ in table 1. Figs. 4 and 5 show that removing the condition arg $\left\{a_{n} a_{n+1}^{*}\right\}= \pm \frac{1}{2} \pi$ increases the maximum achievable redundancy.

In order to record holograms with high diffraction efficiency in Fourier transform holography, it is necessary that $|\mathrm{FT}\{q(x)\}|^{2}$ be rather uniform. This condition can be met if the solutions given in table 1 are

Even function solutions $\left\{a_{n}\right\}_{n=-N}^{N}$ to eqs. (1) which maximize the redundancy parameter $R_{1}$

\begin{tabular}{|c|c|c|c|c|c|}
\hline Entry & $\begin{array}{l}\text { Assumption } \\
\arg \left(a_{n} a_{n+1}^{*}\right)= \pm \frac{1}{2} \pi\end{array}$ & $N$ & $R_{1}$ & $\begin{array}{l}|q(x)|^{2} \\
\text { where } \omega=2 \pi / X\end{array}$ & $\left\{a_{n}\right\}_{n=-N}^{N}$ where $a_{-n}=a_{n}$ \\
\hline A & & 1 & 3 & $1 .+0.66 \cos 2 \omega x$ & $\{i, 1, i\}$ \\
\hline $\mathrm{B}$ & & 2 & 4.25 & 1. $+0.47055 \cos (4 \omega x)$ & $\{\mathrm{i}, 1.00007,-\mathrm{i} 0.50007, .\}$. \\
\hline $\mathrm{C}$ & * & 3 & 4.64 & 1. $+0.43094 \cos (6 \omega x)$ & $\{\mathrm{i}, 1.00007,-\mathrm{i} 0.50007,0.375009, \ldots\}$ \\
\hline $\mathrm{D}$ & & 3 & 5.3 & 1. $+0.37734 \cos (6 \omega x)$ & $\begin{array}{l}\{\mathrm{i}, 0.550074,0.988133-\mathrm{i} 0.151290 \\
-0.633294-\mathrm{i} 0.543546, \ldots\}\end{array}$ \\
\hline $\mathrm{E}$ & $*$ & 4 & 4.99 & $1 .+0.10022 \cos (8 \omega x)$ & $\begin{array}{r}\{\mathrm{i},-1.999953,-\mathrm{i} 1.999907 \\
0.343029,-\mathrm{i} 1.312095, \ldots .\}\end{array}$ \\
\hline F & & 4 & 8.49 & $1 .+0.23231 \cos (8 \omega x)$ & $\begin{array}{l}\{\mathrm{i},-0.999931,0.792831-\mathrm{i} 0.499931 \\
0.621247+\mathrm{i} 0.792776 \\
0.889049+\mathrm{i} 0.181948, \ldots .\}\end{array}$ \\
\hline G & $*$ & 5 & 7.40 & $1 .+0.13162 \cos (10 \omega x)$ & $\begin{array}{l}\{\mathrm{i}, 1.100068,-\mathrm{i} 0.605075 \\
-1.432878, \mathrm{i} 1.393206 \\
-1.433349, \ldots . .\}\end{array}$ \\
\hline $\mathrm{H}$ & * & 6 & 8.47 & $1 .+0.19489 \cos (12 \omega x)$ & $\begin{array}{l}\{\mathrm{i},-1.099933,-\mathrm{i} 0.604926 \\
0.799482, \mathrm{i} 0.696409 \\
0.908429, \mathrm{i} 1.100901, \ldots . .\}\end{array}$ \\
\hline
\end{tabular}




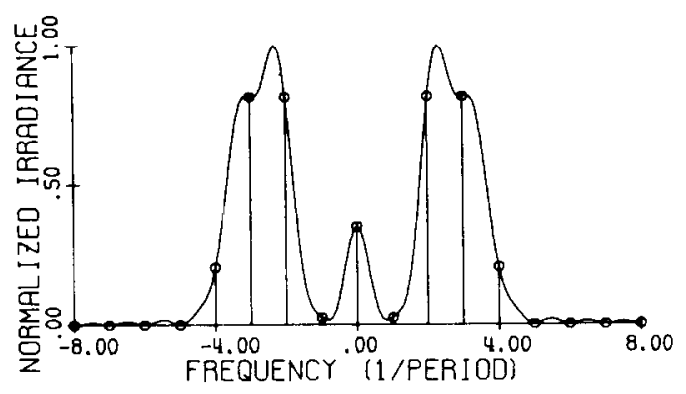

Fig. 4. Power spectrum of $q(x)$ corresponding to entry E of table 1 .

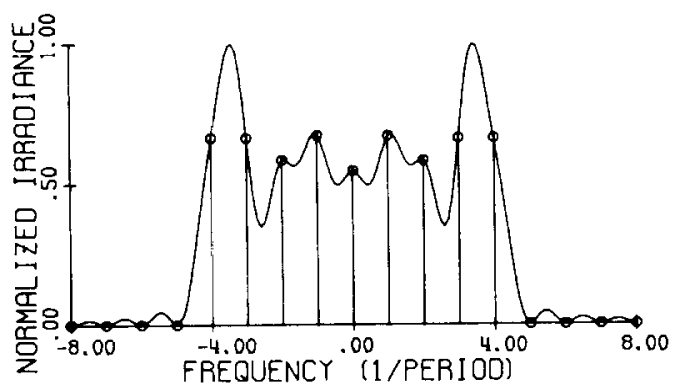

Fig. 5. Power spectrum of $q(x)$ corresponding to entry $\mathrm{F}$ of table 1 .

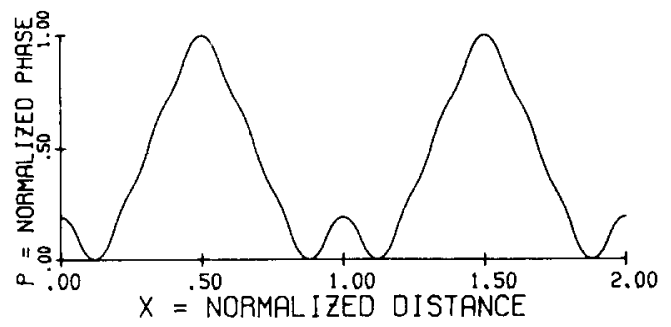

Fig. 6. Normalized phase for $q(x)$ defined by entry F of table 1. $P=1$ corresponds to 1.015229 wavelengths. used in tandem with a coarse lens array to obtain a new modulation function $q(x)=q_{1}(x) q_{2}(x)$, where $q_{1}(x)$ is a solution to eqs. (1) such as entry $F$ of table 1 and $q_{2}(x)$ is a lens array with a period $X_{2}$ large enough to satisfy the equations

FT $\left\{q_{1}(x)\right\}=\sum_{n=-N}^{N} a_{n} \delta\left(f_{x}-n / X_{1}\right)$,

$f_{x}$ is a spatial frequency variable. If

$\left|\mathrm{FT}\left\{q_{2}(x)\right\}\right| \approx \operatorname{rect}\left(X_{1} f_{x}\right)$,

then

$\left|\mathrm{FT}\left\{q_{1}(x) q_{2}(x)\right\}\right|^{2} \approx \operatorname{rect}\left(\frac{X_{1} f_{x}}{2 N+1}\right)$.

Finally, it should be noted that the function $q(x)$ defined by entry $F$ of table 1 appears to meet quite well the redundancy requirements of holographic microfiche systems which store continuous-tone transparencies.

The author wishes to acknowledge many helpful discussions with J. Upatnieks and E.N. Leith.

\section{References}

[1] E.N. Leith and J. Upatnieks, Appl. Opt. 10 (1968) 2085.

[2] A.H. Firester, E.C. Fox, J. Gayeski, W.J. Hannan and M. Lurie, RCA Rev. 33 (1972) 131.

[3] J.R. Klauder, A.C. Price, S. Darlington and W.J. Albersheim, Bell Sy stem Tech, J. 39 (1960) 745.

[4] E.N. Fowle, IEEE Trans. IT-10 (1964) 61.

[5] D. Gabor, IBM J. Res. Develop. 14 (1970) 509. 